100 Chronic HCV patients (63\% males) with mean age of $13 \pm$ 2.8 years in addition to 100 healthy matched controls. Diagnosis of patients relied upon persistently positive HCV PCR for at least 6 months. Eighty two patients received combined pegylated interferon $\alpha 2 \mathrm{~b} 60 \mathrm{mcg} / \mathrm{m}^{2} / \mathrm{w}$ and ribavirin $15 \mathrm{mg} / \mathrm{kg} / \mathrm{d}$. Treatment was continued for 48 weeks for responders (49 patients). All subjects were exposed to history, examination, liver functions, viral markers, HCV PCR and abdominal ultrasound. Liver biopsy was done only for patients. Gene polymorphisms were assessed by restriction fragment length polymorphism.

Results Neither patients nor controls had TLR2 Arg753Gln or TLR4 Asp299Gly polymorphisms. Only 3 patients (3\%) were heterozygous for TLR4 Thr399lle polymorphism without significant difference between patients and controls $(p=0.24)$. No significant correlation was observed between TLR4 Thr399Ile polymorphism and viral load, histologic activity, grade of fibrosis or treatment response ( $\mathrm{p}=0.96,0.21,0.46,0.49$ respectively). Conclusion TLR2 Arg753Gln, TLR4 Asp294Gly and TLR4 Thr399Ile polymorphisms seem to be absent or rare and probably have no role in HCV among Egyptian population.

\section{0-225 LNCRNAS ARE PREFERENTIALLY LOCATED NEAR TRANSCRIPTION FACTORS AND REGULATE EARLY ENDODERM AND LUNG DEVELOPMENT}

${ }^{1} \mathrm{D}$ Swarr, ${ }^{2} \mathrm{M}$ Herriges, ${ }^{3} \mathrm{M}$ Morley, ${ }^{3} \mathrm{M}$ Lu, ${ }^{3} \mathrm{~K}$ Stewart, $2,3,4,5 \mathrm{EE}$ Morrisey . ${ }^{1}$ Div. of Neonatology and Human Genetics, The Children's Hospital of Philadelphia; ' 2 Department of Cell and Developmental Biology; ${ }^{3}$ Department of Medicine; ${ }^{4}$ Institute for Regenerative Medicine; ${ }^{5}$ The Cardiovascular Research Institute, U. of Pennsylvania, Philadelphia, PA

10.1136/archdischild-2014-307384.295

Although long non-coding RNAs (lncRNAs) have been demonstrated to regulate fundamental biologic processes, such as cell proliferation and maintenance of the pluripotent state, their patterns of expression and role during mammalian development remains poorly defined. Using RNA-Seq with a conservative pipeline, we have identified 363 lncRNAs in the lung and

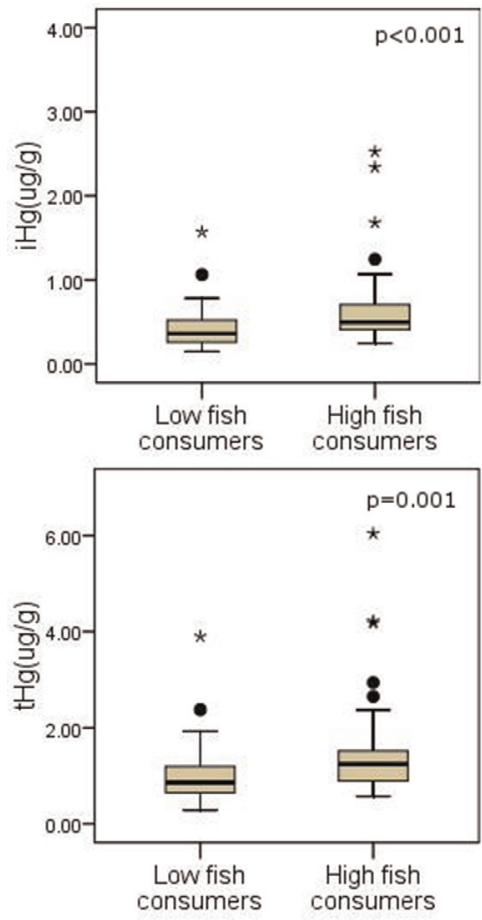

foregut endoderm. These lncRNAs were 3 -fold more likely to be located within $20 \mathrm{~kb}$ of a known transcription factor compared to protein-coding genes. Three lncRNAs were selected for indepth analysis: two lncRNAs adjacent to the critical transcription factors Foxa2 (LncFoxa2) and Nkx2.1 (Nkx2.1-Associated NonCodIng RNA, Nanci), and a novel lncRNA (LL34) with markedly increased expression in embryonic lung. In situ hybridization revealed distinct patterns of expression in the lung for each of these transcripts, with nearly identical patterns of expression between Foxa2 and Nkx2.1 with their neighbouring lncRNAs. Lentiviral shRNA-mediated knockdown of LL34 revealed alterations in the expression of genes involved in early patterning of the foregut and lung, including genes involved in retinoic acid (RA) synthesis, and downstream targets of RA signalling including Foxa1, Gata6, Bmp2, Fgfr2, Fgfr3, and Tgfb3. Most recently, CRISPR-Cas9 technology has been used to generate stable hepatocyte knockout lines of LncFoxa2, which demonstrate that LncFoxa2 may function to regulate endoderm development by both regulation of its neighbouring transcription factor, and also through mechanisms independent of Foxa2. Taken together, these data suggest that lncRNAs play critical roles in the patterning, growth, and differentiation of the foregut and lung.

Funding for this research was provided by NIH 5 U01 HL110942 02 and NIH 5 T32GM008638 17.

\section{Adolescent Health}

\section{PS-001 FISH CONSUMPTION PATTERN AND ITS ASSOCIATION WITH HAIR MERCURY LEVELS IN HONG KONG PRESCHOOL CHILDREN}

${ }^{1}$ PHY Chan, ${ }^{1} \mathrm{HS}$ Lam, ${ }^{1} \mathrm{CLS}$ Chau, ${ }^{2} \mathrm{RCK}$ Cheung, ${ }^{2} \mathrm{MHM}$ Chan, ${ }^{1} \mathrm{AM}$ Li. ${ }^{1}$ Department of Paediatrics, The Chinese University of Hong Kong, Hong Kong, China; ${ }^{2}$ Department of Chemical Pathology, The Chinese University of Hong Kong, Hong Kong, China

\subsection{6/archdischild-2014-307384.296}

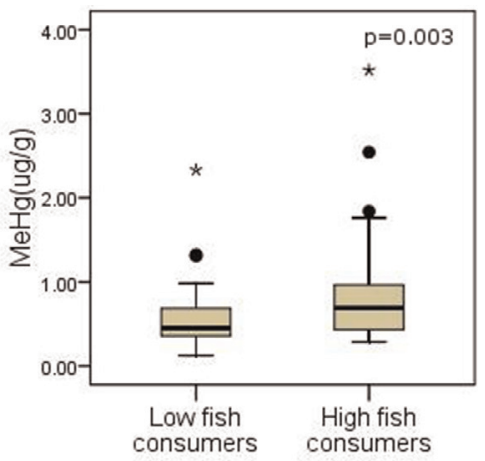

Abstract PS-001 Figure 1 Box plot graphs for low and high fish consumers of their hair iHg, MeHg and tHg MeHg, tHg levels 